\title{
三(芳氧基)芳烃络合低价锔系离子的结构和还原性质理论探索
}

\author{
杨之策 田佳楠 才洪雪李丽 潘清江* \\ (黑龙江大学功能无机材料化学教育部重点实验室 化学化工与材料学院 哈尔滨 150080)
}

\begin{abstract}
摘要 由于具有特殊的电子结构和独特的反应性, 低价钶系配合物已得到广泛关注. 目前, 实验中得到的可分离、晶体 结构确定的 +2 钣系元素配合物少之又少. 本工作通过相对论密度泛函理论探索了低价 $[\mathrm{AnL}]^{z}(\mathrm{An}=\mathrm{Ac} \sim \mathrm{Pu} ; \mathrm{L}=$ $\left[\left({ }^{\mathrm{Me}, \mathrm{Me}} \mathrm{ArOH}\right)_{3} \mathrm{Ar}\right]^{3-} ; z=0$ 和 -1$)$ 的结构和氧化还原性质. 计算发现, 在 $[\mathrm{AnL}] \rightarrow[\mathrm{AnL}]^{-}$还原过程中 $\mathrm{An}-\mathrm{C}_{\mathrm{Ar}}$ 和 $\mathrm{An}-\mathrm{Ar} \mathrm{cent}_{\mathrm{r}}$ 距 离缩短, 这是由于还原电子进入到 $\mathrm{An}$ 和 $\mathrm{Ar}$ 共享区域, 增强了 $\delta(\mathrm{An}-\mathrm{Ar})$ 成键作用所致. 还原时, 配合物 $[\mathrm{AcL}]$ 和 $[\mathrm{ThL}]$ 的 $\mathrm{Ar}$ 基团捕获了大部分还原电子, 而 $\mathrm{Pa} \sim \mathrm{Pu}$ 配合物的则更多地定域在金属周围. 因此, 还原产物中 $\mathrm{Ac}$ 和 $\mathrm{Th}$ 仍为 +3 , 而 $\mathrm{Pa} \sim \mathrm{Pu}$ 则为 +2 、具有 $5 \mathrm{f}^{n}$ 电子组态. 得到的还原电势值随着 $\mathrm{Ac} \sim \mathrm{Pu}$ 整体呈上升趋势, 在 $\mathrm{U}$ 和 $\mathrm{Np}$ 处出现相对 $\mathrm{Pa}$ 和 $\mathrm{Pu}$ 的低点. 这一趋势与电子亲和能和 $\mathrm{An}-\mathrm{C}_{\mathrm{Ar}} / \mathrm{Ar}_{\mathrm{cent}}$ 距离变化有很好的相关性.

关键词 $\delta(\mathrm{An}-\mathrm{Ar})$ 型低价钣系配合物; 三(芳氧基)芳烃配体; 电子结构; 氧化还原性质; 相对论密度泛函理论
\end{abstract}

\section{Theoretical Probe for Tris(aryloxide)arene Complexed Low-valent Actinide Ions and Their Structural/Redox Properties}

\author{
Yang, Zhice Tian, Jianan Cai, Hongxue Li, Li Pan, Qingjiang* \\ (Key Laboratory of Functional Inorganic Material Chemistry of Education Ministry, School of Chemistry and \\ Materials Science, Heilongjiang University, Harbin 150080, China)
}

\begin{abstract}
It is of great significance to identify new oxidation state of actinide, which will enrich actinide coordination chemistry and advance its exploration of chemical bond and reactivity. So far, uranium with $+3 \sim+6$ oxidation states has been widely recognized in complexes. Comparatively, isolated, crystallographically identified U(II) complexes remain rare. Inspired by the pioneering work of Evans and co-workers that $\mathrm{Y} \cdot\left[\mathrm{U}^{\mathrm{II}}\left(\mathrm{Cp}^{\prime}\right)_{3}\right]\left(\mathrm{Y}=[\mathrm{K}(2.2 .2 \text {-cryptand })]^{+}, \mathrm{Cp}^{\prime}=\left[\mathrm{C}_{5} \mathrm{H}_{4} \mathrm{SiMe}_{3}\right]^{-}\right)$ was structurally characterized, several uranium(II) complexes such as $\mathrm{Y} \cdot\left[\mathrm{UL}^{\mathrm{E}}\right]\left(\mathrm{L}^{\mathrm{E}}=\left[\left({ }^{\mathrm{Ad}, \mathrm{Me}} \mathrm{ArO}\right)_{3} \text { mesitylene }\right]^{3-}, \mathrm{Ad}=\right.$ adamantyl), [U(NHAr $\left.\left.{ }^{i \mathrm{Pr} 6}\right)_{2}\right]\left(\mathrm{Ar}^{i \mathrm{Pr} 6}=2,6-\left(2,4,6-{ }^{i} \mathrm{Pr}_{3} \mathrm{C}_{6} \mathrm{H}_{2}\right)_{2} \mathrm{C}_{6} \mathrm{H}_{3}\right), \mathrm{Y} \cdot\left[\mathrm{U}\left\{\mathrm{N}\left(\mathrm{SiMe}_{3}\right)_{2}\right\}_{3}\right]$ and $\left[\mathrm{U}\left(\eta^{5}-\mathrm{C}_{5}{ }^{i} \operatorname{Pr}_{5}\right)_{2}\right]$ were synthetically accessible. Inspection finds that all these $\mathrm{U}(\mathrm{II})$ complexes were prepared in the same route, i.e., utilizing potassium graphite or potassium sphere to reduce respective U(III) parent at low temperature. Cyclopentadiene (Cp) and arene (Ar)-based ligands are involved. They are key to determine U(II) electron configuration, leading to $5 \mathrm{f}^{3} 6 \mathrm{~d}^{1}$ and $5 \mathrm{f}^{4}$, respectively. Moreover, $\delta(\mathrm{U}-\mathrm{Ar})$ bonds play a significant role in stabilizing arene-ligated complexes. With the supporting of Cp-derived ligands, actinide(II) complexes were extended to $\mathrm{Th}, \mathrm{Np}$ and $\mathrm{Pu}$. Unfortunately, it is not the case for the arene ligands, even with massive efforts. Given the prevailing route that actinide(II) complex was synthesized by reducing its trivalent parent, the exploration of redox property will help to guide the synthesis of more novel U(II) and even other actinide(II) complexes. In this respect, theoretical computation based on accurate methodology is greatly appealing. Herein, relativistic density functional theory was exploited to investigate structural and redox properties of $[\mathrm{AnL}]^{z}\left(\mathrm{An}=\mathrm{Ac} \sim \mathrm{Pu} ; \mathrm{L}=\left[\left({ }^{\mathrm{Me}, \mathrm{Me}} \mathrm{ArOH}\right)_{3} \mathrm{Ar}\right]^{3-} ; z=0\right.$ and -1$)$, where analogues of uranium complexes were experimentally known. It is found that the central arene moiety is redox-active for Ac and Th complexes in the reduction reaction, while the metal center is reduced for other complexes. So Ac and Th in reduced products still remain +3 oxidation states, whereas metals in others turn +2 . The $5 \mathrm{f}^{n}$ electronic configuration is unraveled for actinide of $[\mathrm{AnL}]^{-}(\mathrm{An}=\mathrm{Pa} \sim \mathrm{Pu})$, having $3 \sim 6$ electrons, respectively. Calculated redox potential $\left(E^{0}\right)$ increases from $\mathrm{Ac}$ to $\mathrm{Pu}$ in general, where $\mathrm{U}$ and $\mathrm{Np}$ show lower values than adjacent elements. A good correlation has been built between $E^{0}$ and $\Delta\left(\mathrm{An}-\mathrm{C}_{\mathrm{Ar}} / \mathrm{An}-\mathrm{Ar}_{\text {cent }}\right) /$ electron affinity. In brief, the study is expected to provide theoretical support for the synthesis of novel arene-based actinide(II) complexes.
\end{abstract}

Keywords $\delta(\mathrm{An}-\mathrm{Ar})$-type low-valent actinide complexes; tris(aryloxide)arene; electronic structure; redox reaction; relativistic density functional theory

\footnotetext{
*E-mail: panqjitc@163.com

Received July 2, 2020; published August 3, 2020.

Supporting information for this article is available free of charge via the Internet at http://sioc-journal.cn.

Project supported by the National Natural Science Foundation of China (No. 21671060), the Natural Science Foundation of Heilongjiang Province (No. LH2019B029) and the Heilongjiang Touyan Innovation Team Program.

项目受国家自然科学基金(No. 21671060)、黑龙江省自然科学基金(No. LH2019B029)和黑龙江省 “头雁” 团队资助.
} 


\section{1 引言}

氧化态是化学元素最重要的基本性质之一 ${ }^{[1-6]}$. 它 的确定和新价态的延展对研究元素成键和反应性质尤 为重要. 近年, 由于含有 $s, p 、 d 、 f$ 各种类型轨道、外 层电子众多和电子结构极其复杂, 使得锕系元素, 特别 是中部的 $U 、 N p$ 和 $P u$, 具有多种氧化态, 其探索一直是 科学前沿领域的热点课题. 如, Evans 课题组合成了第 一个晶体结构确定、可分离的二价铀配合物 $\mathrm{Y} \cdot\left[\mathrm{U}\left(\mathrm{Cp}^{\prime}\right)_{3}\right]$ $\left(\mathrm{Y}=[\mathrm{K}(2.2 .2 \text {-cryptand })]^{+} \text {和 } \mathrm{Cp}^{\prime}=\left[\mathrm{C}_{5} \mathrm{H}_{4} \mathrm{SiMe}_{3}\right]^{-}\right)^{[7]}$. 随后, 多个课题组先后合成几个二价铀配合物, 包括 $\mathrm{Y} \cdot\left[\mathrm{UL}^{\mathrm{E}}\right]$ $\left(\mathrm{L}^{\mathrm{E}}=\left[\left({ }^{\mathrm{Ad}, \mathrm{Me}} \mathrm{ArOH}\right)_{3} \text { mesitylene }\right]^{3-}, \quad \mathrm{Ad}=\text { adamantyl }\right)^{[8]}$ 、 $\left[\mathrm{U}\left(\mathrm{NHAr}^{\mathrm{iPr} 6}\right)_{2}\right] \quad\left(\mathrm{Ar}^{\mathrm{iPr} 6}=2,6-\left(2,4,6-{ }^{i} \mathrm{Pr}_{3} \mathrm{C}_{6} \mathrm{H}_{2}\right)_{2} \mathrm{C}_{6} \mathrm{H}_{3}\right)^{[9]}$ 、 $\left.\mathrm{Y} \cdot\left[\mathrm{U}\left\{\mathrm{N}\left(\mathrm{SiMe}_{3}\right)_{2}\right\}_{3}\right]\right]^{[10]}$ 和 $\left[\mathrm{U}\left(\eta^{5}-\mathrm{C}_{5}{ }^{i} \mathrm{Pr}_{5}\right)_{2}\right]^{[11]}$. 研究表明, 环戊二烯基(Cyclopentadienyl, Cp)配合物中铀的电子组 态为 $5 f^{3} 6 \mathrm{~d}^{1}$, 而芳香基(Arene, $\mathrm{Ar}$ )的为 $5 \mathrm{f}^{4}\left[\mathrm{Cr}^{[10,12-14]}\right.$. 另外, $\mathrm{Cp}$ 类配体还能稳定 $\mathrm{Th}^{[15]} 、 \mathrm{~Np}^{[16,17]}$ 和 $\mathrm{Pu}^{[18]}$ 二价离子, 形 成的配合物基态电子组态分别为 $6 \mathrm{~d}^{2} 、 5 \mathrm{f}^{3} 6 \mathrm{~d}^{1}$ 和 $5 \mathrm{f}^{6}$. 然 而, 二价钶系金属配合物虽然可以在溶液中获得并得到 可分离的晶体, 但它们即使在低温下仍不稳定, 这给实 验研究其化学反应性带来了极大的挑战. 在这方面, 理 论计算化学为科学工作者深入探索低价锕系配合物提 供了可靠的工具 ${ }^{[3,4,19-25]}$. 如, 石伟群课题组 ${ }^{[19]}$ 在 $\mathrm{Cp}^{\prime}$ 配 位的二价钶系配合物方面的研究工作中, 很好地解释了 已合成配合物的成键和电子性质，同时对实验未合成的 $\mathrm{Pa}$ 和 $\mathrm{Am}$ 配合物的结构和性质给予了理论预测.

相对于环戊二烯基钣系配合物, 目前实验上尚未合 成除铀外芳香基配体配位的二价钶系金属配合物. 受到 Meyer 课题组合成的芳基配合物 $\left[\mathrm{UL}^{\mathrm{E}}\right]^{-}$启发我们计划将 中心金属延展到其它钣系元素 $\mathrm{Ac} \sim \mathrm{Pa} 、 \mathrm{~Np}$ 和 $\mathrm{Pu}$. 配体 结构见图 1. 采用相对论密度泛函理论系统地探索了它 们的几何/电子结构和氧化还原性质, 特别是探讨还原 电势与还原过程中几何参数、电子自旋密度和电子亲和 势变化之间的关联性. 通过总结规律性, 期望为实验合 成更多二价钣系元素配合物提供理论参考.
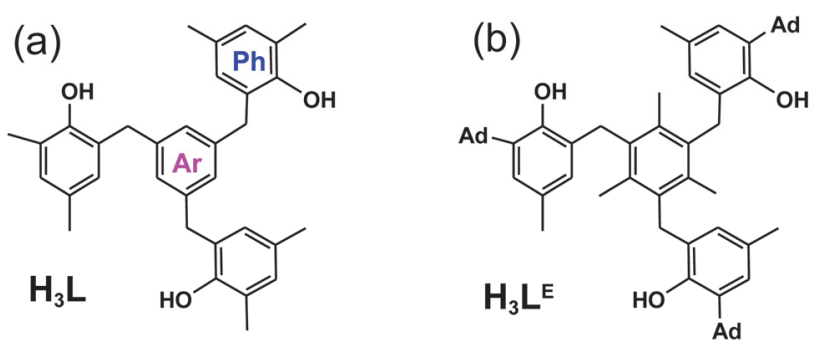

图 1 理论计算 $\mathrm{H}_{3} \mathrm{~L}$ (a)和实验合成 $\mathrm{H}_{3} \mathrm{~L}^{\mathrm{E}}$ (b)配体, 其中中心芳基标记 为 $\mathrm{Ar}$, 其它三个命名为 $3 \mathrm{Ph}$

Figure 1 Ligands of theoretically computed $\mathrm{H}_{3} \mathrm{~L}$ (a) and experimentally synthesized $\mathrm{H}_{3} \mathrm{~L}^{\mathrm{E}}$ (b), where the central arene group is labeled as $\mathrm{Ar}$ and the three arm ones are named as $3 \mathrm{Ph}$

\section{2 结果与讨论}

\section{1 结构性质}

结构优化显示 $\mathrm{An}$ 离子被配体 3 个酚羟基 $\mathrm{O}$ 和中心 $\mathrm{Ar}$ 基团共同配位，其位于 3 个 $\mathrm{O}$ 构成平面(用 $\mathrm{O}_{3}$ 表示) 和 $\mathrm{Ar}$ 平面之间, 见图 2. 其中 $\mathrm{An}$ 偏离 $\mathrm{O}_{3}$ 平面的程度可 以用 $\mathrm{An}-\mathrm{O}_{3}$ 距离和 $\mathrm{O}-\mathrm{An}-\mathrm{O}$ 角描述. 如计算得到 $[\mathrm{AcL}]$ 的 $\mathrm{Ac}-\mathrm{O}_{3}$ 距离为 $0.007 \mathrm{~nm}, \mathrm{Ac}$ 几乎在 $\mathrm{O}_{3}$ 平面内; 对应的 $\mathrm{O}-\mathrm{Ac}-\mathrm{O}$ 角为 $119.9^{\circ}$, 接近 $120^{\circ}$. 而 $[\mathrm{PaL}]^{-}$有最大的 0.077 $\mathrm{nm} \mathrm{Pa}-\mathrm{O}_{3}$ 距离和最小的 $108.7^{\circ} \mathrm{O}-\mathrm{Pa}-\mathrm{O}$ 角. 这一共面性 特征将会在电子结构分析中给予解释. 无论 $[\mathrm{AnL}]$ 还是 $[\mathrm{AnL}]^{-}$, 从 $\mathrm{Ac} \sim \mathrm{U}$, 再从 $\mathrm{U} \sim \mathrm{Pu}, \mathrm{An}-\mathrm{O}$ 键长呈现先逐渐 缩短, 然后增长的规律性. 这与钣系金属中心的共价半 径变化趋势相一致 ${ }^{[26]} . \mathrm{An}-\mathrm{C}_{\mathrm{Ar}}$ 和 $\mathrm{An}-\mathrm{Ar}_{\mathrm{cent}}$ 距离变化基本 保持这一趋势, 细微差别是 $\mathrm{Pa}$ 的距离最短. 简言之, 随 着原子序数增加, 键长的变化在 $\mathrm{Pa}$ 和 $\mathrm{U}$ 处出拐点.
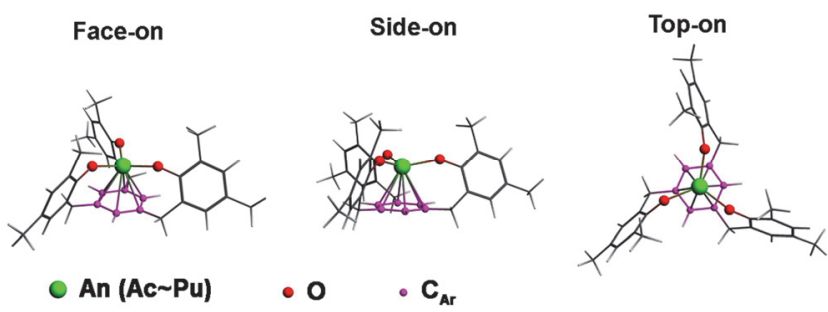

图 2 配合物 $[\mathrm{AnL}]^{z}$ 的结构 $(\mathrm{An}=\mathrm{Ac} \sim \mathrm{Pu} ; z=0$ 和 -1$)$

Figure 2 Structures of $[\mathrm{AnL}]^{z}(\mathrm{An}=\mathrm{Ac} \sim \mathrm{Pu} ; z=0$ and -1$)$

在图 3 中绘制了 $[\mathrm{AnL}]$ 被还原为 $[\mathrm{AnL}]^{-}$的各种几何 参数变化值. 首先, 距离变化 $\Delta\left(A n-C_{A r}\right)$ 和 $\Delta\left(A n-A r_{c e n t}\right)$ 均为负值, 表明还原作用使其变短. 分析电子结构和电 子自旋密度(下面将作具体阐述)发现, 还原电子进入到 $\mathrm{An}$ 和 $\mathrm{Ar}$ 共享区域, 形成 $\delta(\mathrm{An}-\mathrm{Ar})$ 成键作用, 因而缩短 $\mathrm{An}-\mathrm{C}_{\mathrm{Ar}}$ 和 $\mathrm{An}-\mathrm{Ar}_{\mathrm{cent}}$ 距离. 然而, $\mathrm{An}$ 周围定域更多电子使 其原子半径增大, 这会增长 $\mathrm{An}-\mathrm{C}_{\mathrm{Ar}} / \mathrm{Ar}_{\mathrm{cent}}$ 距离. 综合来 看, 两个相反的效应中 $\delta(\mathrm{An}-\mathrm{Ar})$ 成键作用的影响更大, 是导致距离变短的关键因素. 其次, $\Delta\left(A n-\mathrm{C}_{\mathrm{Ar}} / \mathrm{Ar}_{\text {cent }}\right)$ 随 着钶系金属原子序数的增加变得更正, 在 $\mathrm{Pa}$ 和 $\mathrm{Np}$ 处出 现两个拐点, 见图 3(a). 我们推测这一变化趋势与氧化 还原性质相关.

此外, 电子还原使得中心 $\mathrm{Ar}$ 基团上的 $\mathrm{C}_{\mathrm{Ar}}-\mathrm{C}_{\mathrm{Ar}}$ 键长 略微伸长, 即 $\Delta\left(\mathrm{C}_{\mathrm{Ar}}-\mathrm{C}_{\mathrm{Ar}}\right)$ 均为正值. 表明有部分还原电 子进入到 $\mathrm{Ar}$ 区域，破坏了其原来的芳香稳定性. 其中, $\mathrm{Ac}$ 和 $\mathrm{Th}$ 比 $\mathrm{Pa} \sim \mathrm{Pu}$ 增长的多, 因此推测可能有更多的 电子进入到 $[\mathrm{AcL}]^{-}$和 $[\mathrm{ThL}]^{-}$的 $\mathrm{Ar}$ 部分, 这将被后面讨论 的电子自旋密度所证实. $\mathrm{An}-\mathrm{O}$ 和 $\mathrm{An}-\mathrm{O}_{3}$ 距离均伸长, 一 方面由于 $\mathrm{An}$ 部分电子增多, 引起 $\mathrm{An}$ 和 $\mathrm{O}$ 之间的排斥 作用, 另一方面与 An 共价半径增大有关. 还原后角 $\mathrm{O}-\mathrm{An}-\mathrm{O}$ 减小，表明中心金属更偏离 $\mathrm{O}_{3}$ 平面，向着 $\mathrm{Ar}$ 一 侧靠近. 
表 1 优化的 $[\mathrm{AnL}]^{-}$和 $[\mathrm{AnL}]$ 几何参数(键长单位为 $\mathrm{nm}$, 键角单位为 $\left(^{\circ}\right)$ )

Table 1 Optimized geometry parameters for $[\mathrm{AnL}]^{-}$and [AnL] (Distance in nm and angle in $\left(^{\circ}\right)$ )

\begin{tabular}{|c|c|c|c|c|c|c|c|}
\hline Complexes. & Approaches & $\mathrm{An}-\mathrm{C}_{\mathrm{Ar}}{ }^{a}$ & An-Ar ${ }_{\text {cent }}{ }^{b}$ & $\mathrm{An}-\mathrm{O}^{a}$ & $\mathrm{An}-\mathrm{O}_{3}{ }^{c}$ & $\mathrm{C}_{\mathrm{Ar}}-\mathrm{C}_{\mathrm{Ar}}{ }^{a}$ & $\mathrm{O}-\mathrm{An}-\mathrm{O}^{a}$ \\
\hline$[\mathrm{AcL}]^{-}$ & Calc. & 0.2897 & 0.2523 & 0.2400 & 0.0380 & 0.1426 & 117.6 \\
\hline$[\mathrm{ThL}]^{-}$ & Calc. & 0.2654 & 0.2237 & 0.2286 & 0.0622 & 0.1443 & 113.0 \\
\hline$[\mathrm{PaL}]^{-}$ & Calc. & 0.2554 & 0.2111 & 0.2230 & 0.0772 & 0.1440 & 108.7 \\
\hline \multirow[t]{2}{*}[\mathrm{UL}]{$^{-}$} & Calc. & 0.2576 & 0.2143 & 0.2213 & 0.0685 & 0.1431 & 110.9 \\
\hline & Expt. ${ }^{d}$ & 0.2615 & 0.2180 & 0.2236 & 0.0668 & 0.1432 & 111.5 \\
\hline$[\mathrm{NpL}]^{-}$ & Calc. & 0.2595 & 0.2168 & 0.2214 & 0.0655 & 0.1428 & 111.6 \\
\hline$[\mathrm{PuL}]^{-}$ & Calc. & 0.2700 & 0.2298 & 0.2262 & 0.0580 & 0.1418 & 113.8 \\
\hline [AcL] & Calc. & 0.3078 & 0.2737 & 0.2334 & 0.0065 & 0.1408 & 119.9 \\
\hline [ThL] & Calc. & 0.2767 & 0.2373 & 0.2207 & 0.0439 & 0.1425 & 116.2 \\
\hline$[\mathrm{PaL}]$ & Calc. & 0.2658 & 0.2244 & 0.2163 & 0.0554 & 0.1425 & 113.7 \\
\hline \multirow[t]{2}{*}{ [UL] } & Calc. & 0.2692 & 0.2288 & 0.2154 & 0.0479 & 0.1418 & 115.2 \\
\hline & Expt. ${ }^{d}$ & 0.2749 & 0.2350 & 0.2168 & 0.0480 & 0.1433 & 115.3 \\
\hline [NpL] & Calc. & 0.2716 & 0.2319 & 0.2156 & 0.0449 & 0.1415 & 115.8 \\
\hline
\end{tabular}

${ }^{a}$ Average value; ${ }^{b}$ The distance between An and the centroid of arene; ${ }^{c}$ An- $\mathrm{O}_{3}$ denotes the normal distance from the An ion to the plane defined by three oxygen donors. Noting that a positive value means that $\mathrm{An}$ is situated between the $\mathrm{O}_{3}$ and Ar planes. ${ }^{d}$ Experimental values from Ref. [8].
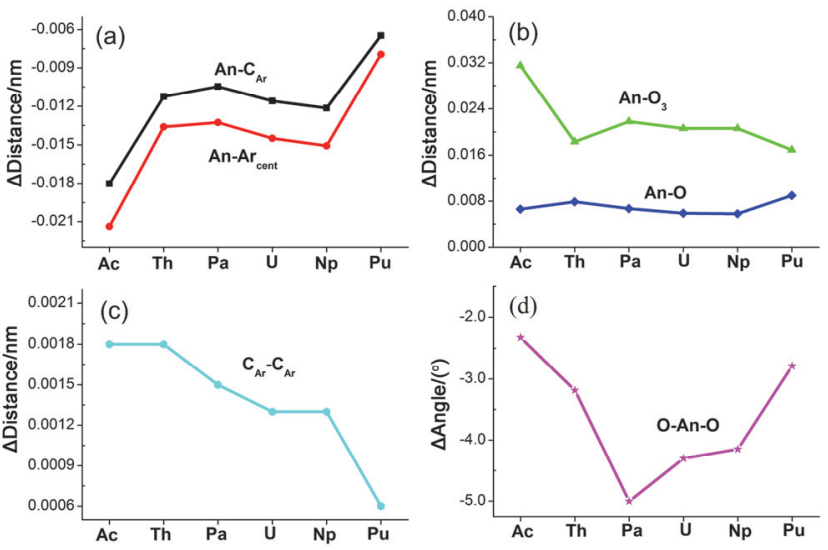

图 $3[\mathrm{AnL}] \rightarrow[\mathrm{AnL}]-$ 还原过程中几何参数变化

Figure 3 Plots of changes of geometry parameters upon reducing [AnL] to $[\mathrm{AnL}]^{-}$

\section{2 电子自旋密度和电子结构}

计算 $[\mathrm{AnL}] \rightarrow[\mathrm{AnL}]^{-}$还原时各个片段的电子自旋密 度变化的平均值 $\left(\Delta S_{\mathrm{Frag}}\right)$ 绘制于图 4 中. 可以直观地看到, 配合物的单电子还原主要发生在 $\mathrm{Ar}$ 或 An 部分, $\Delta S_{\mathrm{Frag}}$ 均超过 $60 \%$; 而 $3 \mathrm{Ph}$ 和 30 的贡献很小, 不超过 5\%, 可 以认为几乎不参与还原反应. 从 $\mathrm{Ac}$ 到 $\mathrm{Pu}$, 配合物的金 属中心部分捕获的电子分别为 $0.13 、 0.36 、 0.65 、 0.80$ 、 0.98 和 1.02, $\mathrm{Ar}$ 部分得到还原电子分别为 $0.82 、 0.60$ 、 $0.31 、 0.15 、-0.01$ 和 -0.10 . 因此, 随原子序数增加, $[\mathrm{AnL}] \rightarrow[\mathrm{AnL}]^{-}$的还原中心逐渐由 $\mathrm{Ar}$ 转变为金属 $\mathrm{An}$. 即, $\mathrm{Ac}$ 和 $\mathrm{Th}$ 配合物还原中心主要为 $\mathrm{Ar}$, 而 $\mathrm{Pa} \sim \mathrm{Pu}$ 的还 原中心主要为金属. 由此, 指认 $[\mathrm{AcL}]^{-}$和 $[\mathrm{ThL}]^{-}$中金属 的价态仍为 +3 , 其配体 $\mathrm{L}^{3-}$ 被还原为 $\mathrm{L}^{4-}$; 而 $[\mathrm{AnL}]^{-}(\mathrm{An}$ $=\mathrm{Pa} \sim \mathrm{Pu}$ )金属中心价态为 +2 .

为理解还原过程中几何结构和电子转移量变化的 规律性, 我们分析总结了配合物在 THF 溶液中的电子
结构. 轨道图和态密度请参见图 $\mathrm{S} 1 \sim \mathrm{S} 14$, 相应的成分 分析见表 $\mathrm{S} 1 \sim \mathrm{S} 12$.

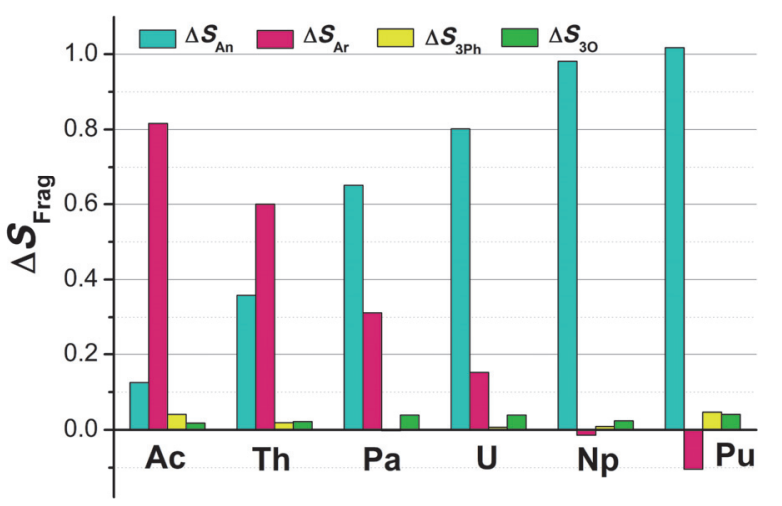

图 $4[\mathrm{AnL}] \rightarrow[\mathrm{AnL}]^{-}$还原过程中配合物的每个片段电子自旋密度变 化 $\left(\Delta \mathrm{S}_{\mathrm{Frag}}\right)$

Figure 4 Difference of electron-spin density of each fragment $\left(\Delta \mathrm{S}_{\text {Frag }}\right)$ upon reducing $[\mathrm{AnL}]$ to $[\mathrm{AnL}]$

轨道成分分析显示, [AcL]的 LUMO 和 L +1 轨道主 要成分均为 $\mathrm{Ar}$, 分别贡献 $81 \%$ 和 $78 \%$, 而能量稍高的 $\mathrm{L}$ +2 轨道有 $90 \%$ 的 Ac 成分. 其占据轨道 $\mathrm{HOMO} \sim \mathrm{H}-5$ 轨道均为 $\pi(3 \mathrm{Ph})$ 轨道特征. 相比之下, $[\mathrm{AcL}]$ 的 $\mathrm{HOMO}$ 轨道中 $\mathrm{Ar}$ 的比重略有下降，为 $75 \%$, 同时有 $9 \%$ 的 $\mathrm{Ac}$ 贡献, 来自于 $6 \mathrm{~d}$ 和 $5 \mathrm{f}$ 成分, 它们轨道重叠共同构成了 $\delta(\mathrm{Ac}-\mathrm{Ar})$ 成键轨道. 轨道具有明显的强极性特征. $[\mathrm{AcL}]^{-}$的能量较低的 $\mathrm{H}-1 \sim \mathrm{H}-5$ 轨道为 $\pi(3 \mathrm{Ph})$ 特征. 同时, 电子结构结果与自旋密度分析和 $\mathrm{Ac}-\mathrm{C}_{\mathrm{Ar}} / \mathrm{Ar}_{\mathrm{cent}}$ 距 离变化相一致. 即, 还原电子被 $\mathrm{Ac}$ 和 $\mathrm{Ar}$ 共享, 但电子 主要定域于 $\mathrm{Ar}$ 基团部分, 形成了强极性 $\delta(\mathrm{Ac}-\mathrm{Ar})$ 键. 因而, $[\mathrm{AcL}]^{-}$中 $\mathrm{Ac}$ 的氧化态基本保持 +3 不变, 而配体 被还原为 $\mathrm{L}^{4-}$; 还原作用使 $[\mathrm{AcL}]^{-}$的 $\mathrm{An}-\mathrm{C}_{\mathrm{Ar}} / \mathrm{Ar}_{\mathrm{cent}}$ 距离比 $[\mathrm{AcL}]$ 的短. 
对于 $\delta(\mathrm{An}-\mathrm{Ar})$ 高占据轨道, 计算得到 $[\mathrm{ThL}]^{-}$的金属 贡献虽有所增加，达到了 $23 \%$, 但仍以 $\mathrm{Ar}$ 为主 $(62 \%)$. 从 $[\mathrm{PaL}]^{-}$开始, 金属的比重在 $40 \% \sim 50 \%$. 而 $[\mathrm{AnL}]^{-}$(An $=\mathrm{U} 、 \mathrm{~Np} 、 \mathrm{Pu})$ 中的金属比重更高, 甚至出现了纯金属的 $\operatorname{An}(5 \mathrm{f})$ 轨道. 由此, 电子结构分析指认 $[\mathrm{AcL}]^{-}$和 $[\mathrm{ThL}]^{-}$中 金属仍保持 +3 的氧化态, 而 $[\mathrm{AnL}]^{-}(\mathrm{An}=\mathrm{Pa} \sim \mathrm{Pu})$ 中金 属为+2. 后者具有 $5 \mathrm{f}^{n}$ 电子组态, 分别对应 $3 \sim 6$ 个电 子. $\left[\mathrm{An}\left(\mathrm{Cp}^{\prime}\right)_{3}\right]^{-}$的研究 ${ }^{[19]}$ 得到 $\mathrm{Pa} \sim \mathrm{Pu}$ 金属的电子组态分 别为 $5 f^{2} 6 d^{1} 、 5 f^{3} 6 d^{1} 、 5 f^{5}$ 和 $5 f^{6}$. 显然, An-Ar 和 An-Cp 相互作用的不同导致这一差异. 在 $[\mathrm{AnL}]^{-}$中, 金属的 $5 \mathrm{f}$ 轨道比 $6 \mathrm{~d}$ 轨道与 $\mathrm{Ar}$ 的 $\pi$ *轨道有更好的对称性匹配, 所 以 $[\mathrm{PaL}]^{-}$和 $[\mathrm{UL}]^{-}$中均为 $5 \mathrm{f}^{n}$ 组态. 对于 $\mathrm{Np}$ 和 $\mathrm{Pu}$ 配合物, 由于 $5 \mathrm{f}$ 轨道的 core-like 特性, 使得 $\mathrm{An}-\mathrm{Ar}$ 和 $\mathrm{An}-\mathrm{Cp}$ 的 差异不再显著, 因而均呈现 $5 \mathrm{f}^{n}$ 电子组态.

分别在表 2 和表 S13 中总结了具有 $\delta(A n-A r)$ 和 $\operatorname{An}(5 \mathrm{f})$ 性质的单电子高占据轨道数目和成分. 可以看出, 无论 $[\mathrm{AnL}]$ 还是 $[\mathrm{AnL}]^{-}$, 随着原子序数增加, $\delta(\mathrm{An}-\mathrm{Ar})$ 轨 道数目先增加、再减少, 且 $\mathrm{Pa} 、 \mathrm{U}$ 和 $\mathrm{Np}$ 的数目一致. $U$ 、 $\mathrm{Np}$ 和 $\mathrm{Pu}$ 配合物中还有纯金属性质的 $\mathrm{An}(5 \mathrm{f})$ 轨道出现, 数目依次增加. 与计算的距离变化 $\Delta\left(A n-C_{A r}\right)$ 和 $\Delta\left(A n-A r_{c e n t}\right)$ 相对照, 发现它们与 $\delta(A n-A r)$ 轨道数目变化 密切相关. 表明 $\delta(\mathrm{An}-\mathrm{Ar})$ 轨道数目是决定 $\mathrm{An}-\mathrm{C}_{\mathrm{Ar}} / \mathrm{Ar}_{\mathrm{cent}}$ 距离缩短的关键因素. 相对而言, $\operatorname{An}(5 \mathrm{f})$ 轨道数目影响 较小. 对比 $[\mathrm{AnL}]$ 和还原产物 $[\mathrm{AnL}]^{-}$则进一步证明上述 观点. 可以看到, 还原产物均比前体有更多的 $\delta(\mathrm{An}-\mathrm{Ar})$ 轨道. 详细分析这些 $\delta(\mathrm{An}-\mathrm{Ar})$ 发现, 这一成键轨道的贡 献中 $\mathrm{Ar}$ 成分从 $\mathrm{Ac}$ 到 $\mathrm{Pu}$ 逐渐减小, 而金属 $\mathrm{An}$ 则呈现上 升趋势(表 S13).

表 2 配合物 $[A n L]^{z}$ 中 $\delta(A n-A r)$ 和 $A n(5 f)$ 单电子占据轨道的数目 Table 2 Number of singly-occupied $\delta(\mathrm{An}-\mathrm{Ar})$ and An(5f) orbitals of $[\mathrm{AnL}]^{z}$

\begin{tabular}{lccccc}
\hline & \multicolumn{2}{c}{$[\mathrm{AnL}]$} & & \multicolumn{2}{c}{$[\mathrm{AnL}]^{-}$} \\
\cline { 2 - 3 } \cline { 5 - 6 } & $\delta(\mathrm{An}-\mathrm{Ar})$ & $\mathrm{An}(5 \mathrm{f})$ & & $\delta(\mathrm{An}-\mathrm{Ar})$ & $\mathrm{An}(5 \mathrm{f})$ \\
\hline $\mathrm{Ac}$ & 0 & 0 & & 1 & 0 \\
$\mathrm{Th}$ & 1 & 0 & & $2^{a}$ & 0 \\
$\mathrm{~Pa}$ & 2 & 0 & & 3 & 0 \\
$\mathrm{U}$ & 2 & 1 & & 3 & 1 \\
$\mathrm{~Np}$ & 2 & 2 & & 3 & 2 \\
$\mathrm{Pu}$ & 0 & 5 & & 2 & 4 \\
\hline
\end{tabular}

${ }^{a}$ Due to its singlet ground state, $[\mathrm{ThL}]^{-}$has doubly-occupied orbitals.

几何分析显示, 所计算的 12 个配合物中 $[\mathrm{AcL}]$ 的金 属和三个 $\mathrm{O}$ 供体原子有最好的共面性 $\left(\mathrm{Ac}-\mathrm{O}_{3} 0.007 \mathrm{~nm}\right)$, 而 $[\mathrm{PaL}]^{-}$中金属偏离 $\mathrm{O}_{3}$ 平面最大 $(0.077 \mathrm{~nm})$. 电子结构 能够很好地解释这一共面性特征. 首先, [AcL]中没有 $\delta(\mathrm{An}-\mathrm{Ar})$ 性质的轨道, 而 $[\mathrm{PaL}]^{-}$中有 3 个, 分别是最少和 最多的(表 2). An 和 Ar 之间成键作用越强, An 会更接近 $\mathrm{Ar}$ 、从而偏离 $\mathrm{O}_{3}$ 平面, $\mathrm{An}$ 和三个 $\mathrm{O}$ 的共面性则越差. 其 次, $[\mathrm{AnL}]^{-}(\mathrm{An}=\mathrm{Pa}, \mathrm{Np}$ 和 $\mathrm{Pu})$ 均具有 3 个 $\delta(\mathrm{An}-\mathrm{Ar})$ 轨道, 对应的 $\mathrm{An}-\mathrm{O}_{3}$ 距离分别为 $0.077 、 0.069$ 和 $0.066 \mathrm{~nm}$. 成
分分析(表 $\mathrm{S} 13$ )表明, [PaL] 的 $\delta$ 轨道中金属和 $\mathrm{Ar}$ 成分比 重相当，而后两个配合物均以金属为主，具有更大的极 性. 所以, 三个配合物中 $\mathrm{Pa}$ 和 $\mathrm{Ar}$ 作用最强, 金属偏离 $\mathrm{O}_{3}$ 平面最大.

采用 $\mathrm{L}^{\mathrm{E}}$ 配体, Meyer 及其合作者合成了 $\mathrm{U}$ 和 $\mathrm{Nd}$ 的 二价和三价配合物, 并尝试了其他稀土元素 ${ }^{[8,27]}$. 如, 实验得到 $\Delta\left(\mathrm{U} / \mathrm{Nd}-\mathrm{Ar}_{\text {cent }}\right)$ 分别为 -0.017 和 $-0.012 \mathrm{~nm}$, 计算得到前者数值为 $-0.011 \mathrm{~nm}$, 这与我们计算的 $U$ 的 $-0.015 \mathrm{~nm}$ 相对应. 我们的研究均指认二价铀为 $5 \mathrm{f}^{4}$ 电 子组态.

\section{3 还原电势}

相对于参比电极 $\mathrm{Fc}^{+} / \mathrm{Fc}$ (二茂铁), 根据如下公式计 算了反应自由能 $\Delta_{\mathrm{r}} G(\mathrm{eV})$ 和恒温恒压可逆条件下的还原 电势 $E^{0}(\mathrm{~V})$ :

$$
\begin{aligned}
& {[\mathrm{AnL}]+\mathrm{Fc} \rightarrow[\mathrm{AnL}]^{-}+\mathrm{Fc}^{+}} \\
& E^{0}=-\Delta_{\mathrm{r}} G / F
\end{aligned}
$$

$F$ 为法拉第常数, 在 $\Delta_{\mathrm{r}} G(\mathrm{sol})$ 中包含 $G_{\mathrm{sol}}$ 贡献, 而 $\Delta_{\mathrm{r}} G(\mathrm{sol}-\mathrm{so})$ 中进一步增加了 $G_{\mathrm{so}}$, 对应地得到 $E^{0}(\mathrm{sol})$ 和 $E^{0}$ (sol-so). 这些数据列于表 3 中, 并绘制了图 5 .
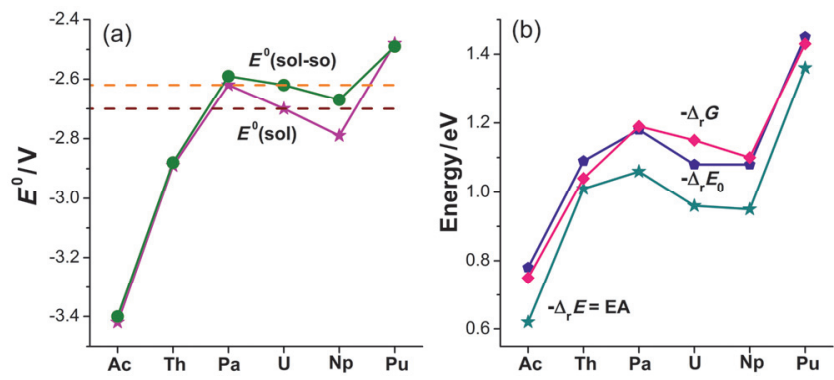

图 5 计算的 $[\mathrm{AnL}] /[\mathrm{AnL}]^{-}$在 $\mathrm{THF}$ 中相对参比电极 $\mathrm{Fc}^{+} / \mathrm{Fc}$ 的还原电势 $\left(\mathrm{V}\right.$ 、左侧), 以及各种反应能量的负值 $\left(\mathrm{eV}\right.$ 、右侧), 其中 $\Delta_{\mathrm{r}} E$ 与电子亲 和势 $\mathrm{EA}$ 互为相反数

Figure 5 Calculated reduction potential $\left(E^{0}\right.$ in $\mathrm{V}$ on the left side) of $[\mathrm{AnL}] /[\mathrm{AnL}]^{-}$relative to the reference electrode $\mathrm{Fc}^{+} / \mathrm{Fc}$ in $\mathrm{THF}$, along with various reaction energies $(\mathrm{eV}$ on the right side) where the minus value of $\Delta_{\mathrm{r}} E$ is equal to the electron affinity (EA)

$E^{0}$ 计算值由 $\mathrm{Ac}$ 到 $\mathrm{Pa}$ 呈单调上升趋势, 在 $\mathrm{Pa}$ 处出 现拐点; 从 $\mathrm{Pa}$ 到 $\mathrm{Np}$ 逐渐下降, $\mathrm{Np}$ 成为低点; 然后到 $\mathrm{Pu}$ 又再次上升. 其大小顺序为 $\mathrm{Pu}>\mathrm{Pa}>\mathrm{U}>\mathrm{Np}>\mathrm{Th}>\mathrm{Ac}$. 两种计算级别的还原电势 $E^{0}(\mathrm{sol})$ 和 $E^{0}(\mathrm{sol}-\mathrm{so})$ 有完全一 致的趋势. 例如，在同时考虑溶剂化效应和旋-轨耦合 效应时, $\mathrm{Ac} \sim \mathrm{Pu}$ 的还原电势值分别为 $-3.40 、 2.88$ 、 $-2.59 、-2.62 、-2.67$ 和 $-2.49 \mathrm{~V}$. 我们计算铀的 -2.62 $\mathrm{V}$ 数值与实验测得的 $-2.50 \mathrm{~V}$ 相接近, 表明使用的计算 方法适用于 $\mathrm{Ar}$ 类配体配位的铜系体系. 目前, 实验已经 通过还原三价铀母体成功制备了二价铀配合物 ${ }^{[8]}$. 如果 以 $\mathrm{U}$ 作为参考和单从还原电势数值考量, $\mathrm{Pu}$ 和 $\mathrm{Pa}$ 具有 更正的还原电势，是可能通过还原母体配合物制备得到 二价产物的. 
表 3 计算的 $[\mathrm{AnL}] /[\mathrm{AnL}]^{-}$单电子还原反应能以及在 $\mathrm{THF}$ 中的还原电势 $E^{0}\left(\mathrm{~V}\right.$, 参比电极 $\left.\mathrm{Fc}^{+} / \mathrm{Fc}\right)$

Table 3 Energies $(\mathrm{eV})$ of the single-electron reduction reactions of complexes from $[\mathrm{AnL}]$ to $[\mathrm{AnL}]^{-}$, along with reduction potential $E^{0}\left(\mathrm{~V}\right.$, versus $\left.\mathrm{Fc}{ }^{+} / \mathrm{Fc}\right)$ in THF

\begin{tabular}{llllllll}
\hline $\mathrm{An}$ & $\Delta_{\mathrm{r}} E^{a}$ & $\Delta_{\mathrm{r}} E_{0}{ }^{a}$ & $\Delta_{\mathrm{r}} G(\mathrm{gas})^{a}$ & $\Delta_{\mathrm{r}} G(\mathrm{sol})^{b}$ & $\Delta_{\mathrm{r}} G(\mathrm{sol}-\mathrm{so})^{b}$ & $E^{0}(\mathrm{sol})^{c}$ & $E^{0}(\mathrm{sol}-\mathrm{so})^{c}$ \\
\hline $\mathrm{Ac}$ & -0.62 & -0.78 & -0.75 & -1.57 & -1.59 & -3.42 & -3.40 \\
$\mathrm{Th}$ & -1.01 & -1.09 & -1.04 & -2.10 & -2.10 & -2.89 & -2.88 \\
$\mathrm{~Pa}$ & -1.06 & -1.18 & -1.19 & -2.37 & -2.39 & -2.62 & -2.59 \\
$\mathrm{U}$ & -0.96 & -1.08 & -1.15 & -2.29 & -2.37 & -2.70 & -2.62 \\
$\mathrm{~Np}$ & -0.95 & -1.08 & -1.10 & -2.20 & -2.32 & -2.79 & -2.67 \\
$\mathrm{Pu}$ & -1.36 & -1.45 & -1.43 & -2.51 & -2.50 & -2.48 & -2.49 \\
\hline
\end{tabular}

${ }^{a}$ The total energy $\left(\Delta_{\mathrm{r}} E\right)$, total energy including zero-point vibrational energy $\left(\Delta_{\mathrm{r}} E_{0}\right)$ and free energy $\Delta_{\mathrm{r}} G($ gas $)$ in the gas phase for the single-electron reduction reactions of actinide complexes. ${ }^{b}$ The solvation energy is included in $\Delta_{\mathrm{r}} G(\mathrm{sol})$, and both solvation and spin-orbit energies are added in $\Delta_{\mathrm{r}} G($ sol-so). These energies were calculated by the ADF code. ${ }^{c}$ The free energies, both $\Delta_{\mathrm{r}} G(\mathrm{sol})$ and $\Delta_{\mathrm{r}} G(\mathrm{sol}-\mathrm{so})$, for the reference electrode of $\mathrm{Fc}^{+} / \mathrm{Fc}$ were calculated to be $-4.99 \mathrm{eV}$. Thus, reduction potentials, $E^{0}$ (sol) and $E^{0}$ (sol-so), were obtained according to Eq. (2).

与结构性质对比发现， $E^{0}$ 的变化趋势与 $[\mathrm{AnL}] \rightarrow[\mathrm{AnL}]^{-}$还原过程中 $\Delta\left(\mathrm{An}-\mathrm{C}_{\mathrm{Ar}}\right)$ 和 $\Delta\left(\mathrm{An}-\mathrm{Ar}_{\mathrm{cent}}\right)$ 的相 似(图 3(a)和图 5(a)). 同时, 还与电子自旋密度变化 $\left(\Delta S_{\mathrm{An}}\right.$ 和 $\Delta S_{\mathrm{Ar}}$ )趋势有很好的相关性(图 4). 值得指出的 是, $E^{0}$ 趋势和 $[\mathrm{AnL}]^{-}$中 $\delta(\mathrm{An}-\mathrm{Ar})$ 成键轨道的数目变化有 相关性. 更为直接的相关性可与反应能量比较得到. 如图 5(b) 所示, $\Delta_{\mathrm{r}} G 、 \Delta_{\mathrm{r}} E^{0}$ 和 $\Delta_{\mathrm{r}} E$ 的变化趋势与 $E^{0}$ 的基本 一致. 我们知道 $\Delta_{\mathrm{r}} E$ 与电子亲和能(EA)互为相反数. 而 EA 是配位化学和材料科学经常采用的, 衡量配合物得 到电子难易的重要指标. 因而, 建立的 $\mathrm{EA}$ 和 $E^{0}$ 正相关 性可以为钣系氧化还原研究提供支持[28,29].

\section{3 结论}

计算的 $[\mathrm{AnL}] /[\mathrm{AnL}]^{-}$还原电势 $\left(E^{0}\right)$ 数值顺序为 $\mathrm{Pu}>$ $\mathrm{Pa}>\mathrm{U}>\mathrm{Np}>\mathrm{Th}>\mathrm{Ac}$. 其中, 铀配合物的 $-2.62 \mathrm{~V}$ 值与 实验测得的 $-2.50 \mathrm{~V}$ 相接近. 由于实验已通过还原三价 铀母体配合物成功合成二价铀配合物, 如果以 $E^{0}$ 作为 参考标准, 具有更正 $E^{0}$ 的二价 $\mathrm{Pu}$ 和 $\mathrm{Pa}$ 配合物很可能通 过同样实验合成路线获得. 发现 $E^{0}$ 变化趋势与还原过 程中距离变化 $\Delta\left(\mathrm{An}-\mathrm{C}_{\mathrm{Ar}}\right)$ 和 $\Delta\left(\mathrm{An}-\mathrm{Ar}_{\mathrm{cent}}\right)$ 、电子自旋密度 差值 $\Delta S_{\mathrm{An}}$ 和 $\Delta S_{\mathrm{Ar}}$ 以及电子亲和势 (EA)均有很好的相关 性; 而且, $[\mathrm{AnL}]^{-}$中 $\delta(\mathrm{An}-\mathrm{Ar})$ 性质单电子占据轨道数目 有相似的变化规律. $\delta(\mathrm{An}-\mathrm{Ar})$ 成键轨道数目的增多是 导致 $\mathrm{An}-\mathrm{C}_{\mathrm{Ar}} / \mathrm{Ar}_{\text {cent }}$ 距离缩短的关键因素. $\mathrm{Ac}$ 和 $\mathrm{Th}$ 配合物 被还原时电子主要被 $\mathrm{Ar}$ 基团捕获，而 $\mathrm{Pa} \sim \mathrm{Pu}$ 配合物的 则主要被金属 $\mathrm{An}$ 获得. 因此, 还原产物中 $\mathrm{Ac}$ 和 Th 仍 为 +3 , 而 $\mathrm{Pa} \sim \mathrm{Pu}$ 则为 +2 . 指认 $[\mathrm{AnL}]^{-}(\mathrm{An}=\mathrm{Pa} \sim \mathrm{Pu})$ 的金属电子组态为 $5 \mathrm{f}^{n}$ 构型, 分别对应 $3 \sim 6$ 个电子. 总 之, 本工作研究有望为合成新型 $\mathrm{Ar}$ 基二价锕系配合物 提供理论参考, 同时为非水溶液低价钣系配位化学发展 提供基础数据.

\section{4 计算方法}

系统探索了一系列钶系配合物 $[\mathrm{AnL}]^{z}(\mathrm{An}=\mathrm{Ac} \sim$ $\mathrm{Pu} ; \mathrm{L}=\left[\left({ }^{\mathrm{Me}, \mathrm{Me}} \mathrm{ArOH}\right)_{3} \mathrm{Ar}\right]^{3-} ; z=0$ 和 1). 为节省计算资源,
使用简化的 $\mathrm{L}$ 配体代替实验的 $\mathrm{L}^{\mathrm{E}}$. 它们的结构如图 1 所 示. 考虑到配合物的电子在轨道中的可能排布, 对它们 的各种电子自旋态异构体进行了结构优化. 其中 $[\mathrm{AcL}]^{-} 、[\mathrm{AcL}]$ 和 $[\mathrm{ThL}]$ 只考察了一种自旋异构体, 而其 它配合物的异构体间相对能量列于补充材料的表 S14 中. 本工作的分析和讨论将重点关注基态异构体. 另外, 我们重点讨论了 $\alpha$ 自旋轨道, 而对于不是高自旋基态体 系( 如 $\left.[\mathrm{PaL}]^{-}\right)$, 还给出了 $\beta$ 自旋轨道图和相应的成分数 据. 为方便讨论, 除非特殊说明, HOMO、 $\mathrm{H}-1$ 等均指 $\alpha$ 自旋单电子占据轨道.

采用 Priroda 程序 $(6.0 \text { 版 })^{[30]}$ 进行配合物的结构优化, 且未对其进行对称性限制, 体系电子态为 ${ }^{2 \mathrm{~S}+1} \mathrm{~A}$. 使用 标量相对论全电子(AE)哈密顿、广义梯度近似的 PBE 泛函 ${ }^{[31]}$ 和全电子高斯基组 ${ }^{[32]}$. 具体基组为 An (34s33p24d18f6g)/(10s9p7d4f1g)、O (10s7p3d)/(3s2p1d)、 C $(10 \mathrm{~s} 7 \mathrm{p} 3 \mathrm{~d}) /(3 \mathrm{~s} 2 \mathrm{p} 1 \mathrm{~d})$ 和 $\mathrm{H}(6 \mathrm{~s} 2 \mathrm{p}) /(2 \mathrm{~s} 1 \mathrm{p})$. 随后的频率计 算没有得到虚频, 表明每个结构对应势能面的极小值 点. 同时, 还得到了零点振动能 $(\mathrm{ZPVE})$ 、熵 $(S)$ 、焓 $(H)$ 和自由能 $(G)$ 等热力学函数. 文中仅进行了单参考态 DFT 计算, 并未考虑多组态效应.

基于优化的结构, 运用 $\mathrm{ADF}$ 程序 ${ }^{[33]}$ 计算了配合物 在 THF 溶液中的电子结构、溶剂化能 $\left(G_{\mathrm{sol}}\right)$ 和自旋-轨道 耦合能 $\left(G_{\mathrm{so}}\right)$. 积分格点参数选用 $6.0 * 6.0 * 6.0$. 应用 COSMO 模型 ${ }^{[34]}$ 模拟 THF 溶剂的环境效应, 其介电常数 为 7.58. 采用标量和旋-轨耦合相对论的 ZORA 哈密顿、 $\mathrm{PBE}$ 泛函和 Slater 型 TZP 基组, 其中冻结铀 $1 \mathrm{~s} \sim 4 \mathrm{f}$ 的 60 个内核电子, 对外层 32 个电子进行全电子计算.

\section{References}

[1] Wang, X.; Liu, Y., Nuclear Chemistry and Radiochemistry, Beijing University Press, 2007 (in Chinese). (王祥云, 刘元方, 核化学与放 射化学, 北京大学出版社, 北京, 2007.)

[2] Schaedle, D.; Anwander, R. Chem. Soc. Rev. 2019, 48, 5752.

[3] Wang, D.; van Gunsteren, W. F.; Chai, Z. Chem. Soc. Rev. 2012, 41, 5836.

[4] Shi, W.-Q.; Yuan, L.-Y.; Wang, C.-Z.; Wang, L.; Mei, L.; Xiao, C.-L.; Zhang, L.; Li, Z.-J.; Zhao, Y.-L.; Chai, Z.-F. Adv. Mater. 2014, 26, 7807.

[5] Wang, N.; Pang, H.; Yu, S.; Gu, P.; Song, S.; Wang, H.; Wang, X. 
Acta Chim. Sinica 2019, 77, 143 (in Chinese). (王宁, 庞宏伟, 于淑 君，顾鹏程，宋爽，王宏青，王祥科，化学学报，2019，77，143.)

[6] Yue, G.; Gao, R.; Zhao, P.; Chu, M.; Shuai, M. Acta Chim. Sinica 2016, 74, 657 (in Chinese). (岳国宗, 高瑞, 赵鹏翔, 褚明福, 帅茂 兵, 化学学报, 2016, 74, 657.)

[7] MacDonald, M. R.; Fieser, M. E.; Bates, J. E.; Ziller, J. W.; Furche, F.; Evans, W. J. J. Am. Chem. Soc. 2013, 135, 13310.

[8] La Pierre, H. S.; Scheurer, A.; Heinemann, F. W.; Hieringer, W.; Meyer, K. Angew. Chem. Int. Ed. 2014, 53, 7158.

[9] Billow, B. S.; Livesay, B. N.; Mokhtarzadeh, C. C.; McCracken, J.; Shores, M. P.; Boncella, J. M.; Odom, A. L. J. Am. Chem. Soc. 2018, 140, 17369.

[10] Ryan, A. J.; Angadol, M. A.; Ziller, J. W.; Evans, W. J. Chem. Commun. 2019, 55, 2325.

[11] Guo, F.-S.; Tsoureas, N.; Huang, G.-Z.; Tong, M.-L.; Mansikkamaeki, A.; Layfield, R. A. Angew. Chem. Int. Ed. 2020, 59, 2299.

[12] Windorff, C. J.; MacDonald, M. R.; Meihaus, K. R.; Ziller, J. W.; Long, J. R.; Evans, W. J. Chem. Eur. J. 2016, 22, 772.

[13] Moehring, S. A.; Evans, W. J. Chem. Eur. J. 2020, 26, 1530.

[14] Huh, D. N.; Ziller, J. W.; Evans, W. J. Inorg. Chem. 2018, 57, 11809.

[15] Langeslay, R. R.; Fieser, M. E.; Ziller, J. W.; Furche, F.; Evans, W. J. Chem. Sci. 2015, 6, 517.

[16] Su, J.; Windorff, C. J.; Batista, E. R.; Evans, W. J.; Gaunt, A. J.; Janicke, M. T.; Kozimor, S. A.; Scott, B. L.; Woen, D. H.; Yang, P. J. Am. Chem. Soc. 2018, 140, 7425.

[17] Dutkiewicz, M. S.; Apostolidis, C.; Walter, O.; Arnold, P. L. Chem. Sci. 2017, 8, 2553.

[18] Windorff, C. J.; Chen, G. P.; Cross, J. N.; Evans, W. J.; Furche, F.; Gaunt, A. J.; Janicke, M. T.; Kozimor, S. A.; Scott, B. L. J. Am. Chem. Soc. 2017, 139, 3970.

[19] Wu, Q. Y.; Lan, J. H.; Wang, C. Z.; Cheng, Z. P.; Chai, Z. F.; Gibson, J. K.; Shi, W. Q. Dalton Trans. 2016, 45, 3102.

[20] Chen, F.; Qu, N.; Wu, Q.; Zhang, H.; Shi, W.; Pan, Q. Acta Chim. Sinica 2017, 75, 457 (in Chinese). (陈方园, 曲宁, 吴群燕, 张红 星, 石伟群, 潘清江, 化学学报, 2017, 75, 457.)

[21] Zhao, S.; Zhong, Y.; Guo, Y.; Zhang, H.; Pan, Q. Acta Chim. Sinica 2016, 74, 683 (in Chinese). (赵思魏, 钟宇䂀, 郭元茹, 张红星, 潘 清江, 化学学报, 2016, 74, 683.)

[22] Zhang, X.; Wang, Y.; Morales-Martinez, R.; Zhong, J.; de Graaf, C.;
Rodriguez-Fortea, A.; Poblet, J. M.; Echegoyen, L.; Feng, L.; Chen, N. J. Am. Chem. Soc. 2018, 140, 3907.

[23] Ding, W.; Liu, Y.; Wang, D. Chem. Eur. J. 2018, 24, 19289.

[24] Hu, H.-S.; Wei, F.; Wang, X.; Andrews, L.; Li, J. J. Am. Chem. Soc. 2014, 136, 1427.

[25] Wang, Y.-L.; Hu, H.-S.; Li, W.-L.; Wei, F.; Li, J. J. Am. Chem. Soc. 2016, $138,1126$.

[26] Pyykko, P. J. Phys. Chem. A 2015, 119, 2326.

[27] Fieser, M. E.; Palumbo, C. T.; La Pierre, H. S.; Halter, D. P.; Voora, V. K.; Ziller, J. W.; Furche, F.; Meyer, K.; Evans, W. J. Chem. Sci. 2017, 8, 7424.

[28] Lewis, A. J.; Carroll, P. J.; Schelter, E. J. J. Am. Chem. Soc. 2013, 135,13185

[29] Elkechai, A.; Mani, Y.; Boucekkine, A.; Ephritikhine, M. Inorg. Chem. 2012, 51, 6943.

[30] Laikov, D. N.; Ustynyuk, Y. A. Russ. Chem. Bull. 2005, 54, 820.

[31] Perdew, J. P.; Burke, K.; Ernzerhof, M. Phys. Rev. Lett. 1996, 77, 3865.

[32] Laikov, D. N. Chem. Phys. Lett. 2005, 416, 116.

[33] Baerends, E. J.; Ziegler, T.; Autschbach, J.; Bashford, D.; Bérces, A. Bickelhaupt, F. M.; Bo, C.; Boerrigter, P. M.; Cavallo, L.; Chong, D. P.; Deng, L.; Dickson, R. M.; Ellis, D. E.; van Faassen, M.; Fan, L.; Fischer, T. H.; Fonseca Guerra, C.; Franchini, M.; Ghysels, A.; Giammona, A.; van Gisbergen, S. J. A.; Götz, A. W.; Groeneveld, J. A.; Gritsenko, O. V.; Grüning, M.; Gusarov, S.; Harris, F. E.; van den Hoek, P.; Jacob, C. R.; Jacobsen, H.; Jensen, L.; Kaminski, J. W.; van Kesse, G.; Kootstra, F.; Kovalenko, A.; Krykunov, M. V.; van Lenthe, E.; McCormack, D. A.; Michalak, A.; Mitoraj, M.; Morton, S. M.; Neugebauer, J.; Nicu, V. P.; Noodleman, L.; Osinga, V. P.; Patchkovskii, S.; Pavanello, M.; Philipsen, P. H. T.; Post, D.; Pye, C. C.; Ravenek, W.; Rodríguez, J. I.; Ros, P.; Schipper, P. R. T.; van Schoot, H.; Schreckenbach, G.; Seldenthuis, J. S.; Seth, M.; Snijders, J. G.; Solà, M.; Swart, M.; Swerhone, D.; te Velde, G.; Vernooijs, P.; Versluis, L.; Visscher, L.; Visser, O.; Wang, F.; Wesolowski, T. A.; van Wezenbeek, E. M.; Wiesenekker, G.; Wolff, S. K.; Woo, T. K.; Yakovlev, A. L., ADF (2014.06 version), SCM, Theoretical Chemistry, Vrije Universiteit, Amsterdam, The Netherlands, 2014.

[34] Klamt, A.; Schuurmann, G. J. Chem. Soc., Perkin Trans. 1993, 799.

(Cheng, B.) 